\title{
Sox5 and Th17 cell differentiation
}

Editorial

\section{Akira Suto, Shigeru Tanaka and Hiroshi Nakajima}

Sox (SRY-related high-mobility-group (HMG)-box) transcription factors, which are comprised of 20 genes containing a conserved HMG DNA-binding domain, are divided into 8 groups (from $\mathrm{A}$ to $\mathrm{H}$ ) according to structural homologies [reviewed in 1-2]. SoxC group, which is composed of Sox4, Sox11, and Sox12, has a HMG box domain and a transactivation domain and plays important roles in the development of heart, nerve system, kidney, and pancreas. SoxD group, which is composed of Sox5, Sox6, and Sox13, has a HMG box domain in C-terminal half and group-specific coiled-coil domain(s) in N-terminal half. SoxD proteins interact with each other through their coiled-coil domain and their activity is likely to be influenced by other molecules with which they interact. Sox 5 and Sox6 are paralogous genes highly expressed in spermatids, neurons, oligodendrocytes, and chondrocytes, and are indispensable for chondrogenesis.

Regarding the roles of Sox family genes in $\mathrm{T}$ cell immunity, it has been demonstrated that Sox 4 suppresses GATA3 function and thereby inhibits Th2 cell-mediated inflammation [reviewed in 3]. On the other hand, Sox 13 plays a critical role in the development of $\gamma \delta \mathrm{T}$ cell receptor $^{+}$thymocytes and Sox13, together with Sox4, induces the differentiation of IL-17-producing $\gamma \delta \mathrm{T}$ cells ( $\mathrm{T} \gamma \delta 17$ cells) through the induction of orphan nuclear receptor ROR $\gamma \mathrm{t}$ [3]. Sox 4 is also expressed in $\alpha \beta \mathrm{T}$ cells; however, Sox4 is not essential for the differentiation of IL-17-producing $\alpha \beta \mathrm{T}$ cells (Th17 cells) [3], which are involved not only in the host defense against extracellular pathogens but also in the pathogenesis of autoimmune diseases [4]. Because ROR $\gamma \mathrm{t}$, which is encoded by Rorc gene, plays a central role in the differentiation of Th17 cells as well as T $\gamma \delta 17$ cells [4], it is suggested that the involvement of Sox transcription factors in ROR $\gamma \mathrm{t}$ induction seems different between Th17 cells and T $\gamma \delta 17$ cells.

It is well established that IL-6- and/or IL-21mediated Stat3 activation is indispensable for the induction of ROR $\gamma \mathrm{t}$ during Th17 cell differentiation. Stat3 binds to intron 1 of Rorc gene and induces chromatin remodeling of the locus. In addition, Stat 3 activation results in the induction of many genes implicated in Th17 cell differentiation such as Nfkbiz, Rora, Batf, Irf4, Ahr, Maf, and HIF-1 $\alpha$ (Figure 1) [4]. Among these genes, HIF1 has been shown to activate Rorc promoter. However, the downstream targets of Stat3 for ROR $\gamma$ t induction in Th17 cells have not been fully understood. To identify the downstream pathway of IL-6-Stat3 signaling for Th17 cell differentiation, we have performed DNA microarray analysis of IL-6-stimulated $\mathrm{CD}^{+} \mathrm{T}$ cells. We found that c-Maf and a novel isoform of Sox 5 (named Sox 5 t) were highly induced in IL-6-stimulated $\mathrm{CD} 4^{+} \mathrm{T}$ cells and that

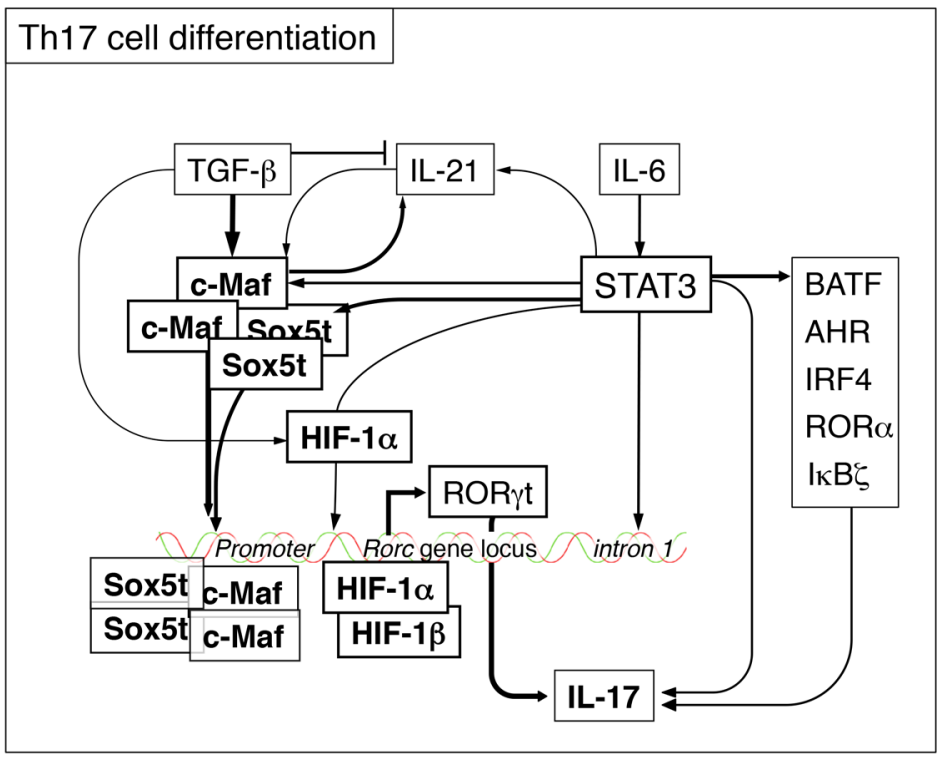

Figure 1: A schematic model for the role of Sox5t and c-Maf in Th17 cell differentiation 
Stat3 was indispensable for the induction of c-Maf and Sox 5t [5]. We also found that severity of Th17 cellmediated immune responses, such as experimental autoimmune encephalomyelitis and delayed-type hypersensitivity, were reduced in T cell-specific Sox5deficient mice [5]. Moreover, Th17 cell differentiation was impaired in T cell-specific Sox5-deficient mice both in vivo and in vitro, indicating that Sox $5 \mathrm{t}$ is involved in Th17 cell differentiation.

To address the mechanism underlying Sox 5tmediated Th17 cell differentiation, we examined the effect of retrovirus-mediated Sox $5 \mathrm{t}$ induction on Th17 cell differentiation. Intriguingly, although the enforced expression of Sox $5 \mathrm{t}$ itself did not induce IL-17 production in $\mathrm{CD}^{+} \mathrm{T}$ cells, the expression of Sox $5 \mathrm{t}$ together with c-Maf significantly induced IL-17 production even under neutral conditions [5]. Co-induction of Sox 5t and c-Maf induced IL-17 production in Stat3-deficient CD4 ${ }^{+} \mathrm{T}$ cells but not in ROR $\gamma$ t-deficient $\mathrm{CD}^{+} \mathrm{T}$ cells, suggesting that Sox 5t and c-Maf induce Th17 cell differentiation as downstream effectors of Stat 3 and as upstream inducers of ROR $\gamma \mathrm{t}$. We further demonstrated that ROR $\gamma \mathrm{t}$ was one of the direct targets of Sox $5 \mathrm{t}$ and c-Maf by integrating the data of RNA-seq analyses and ChIP-seq analyses of Sox5t- and c-Maf-expressed CD4 ${ }^{+} \mathrm{T}$ cells. Moreover, we confirmed that Sox $5 \mathrm{t}$ and c-Maf activated ROR $\gamma \mathrm{t}$ promoter in primary $\mathrm{CD}^{+} \mathrm{T}$ cells. Analyses using deletion mutants of Sox $5 \mathrm{t}$ and c-Maf revealed that Sox $5 \mathrm{t}$ was associated with c-Maf via HMG domain of Sox $5 t$ and DNA-binding domain of c-Maf.

Our study has demonstrated that Sox $5 t$ is expressed in Th17 cells and together with c-Maf, induces ROR $\gamma \mathrm{t}$ expression during Th17 cell differentiation (Figure
1). With regard to the relationship between Sox 5 and autoimmune diseases in humans, it has been shown that Sox 5 is one of the most strikingly upregulated transcription factors in whole blood in patients with multiple sclerosis [6]. In addition, a genome-wide association study (GWAS) has revealed that Sox 5 is associated with limited systemic sclerosis [7]. Taken together, these results suggest the involvement of Sox 5 expressed in $\mathrm{CD}^{+} \mathrm{T}$ cells in the pathogenesis of autoimmune diseases through the induction of Th17 cell differentiation.

Hiroshi Nakajima: Department of Allergy and Clinical Immunology, Graduate School of Medicine, Chiba University, Chiba, Japan

Correspondence to: Hiroshi Nakajima, email nakajimh@faculty.chiba-u.jp

Keywords: Immunology and Microbiology Section, Immune response, Immunity

Received: June 24, 2015

Published: July 03, 2015

\section{REFERENCES}

1. Lefebvre V, et al. Int J Biochem Cell Biol. 2007; 39: 2195214.

2. Wegner M. Int J Biochem Cell Biol. 2010; 42: 381-90.

3. Kang J, et al. Annu Rev Immunol. 2015; 33: 505-38.

4. Ghoreschi K, et al. Trends Immunol. 2011; 32: 395-401.

5. Tanaka S, et al. J Exp Med. 2014; 211: 1857-74.

6. Riveros C, et al., PLoS One. 2010; 5: e14176.

7. Gorlova O, et al., PLoS Genet. 2011; 7: e1002178. 